\title{
Measurement of the Current-Voltage Curve of Photovoltaic Cells Based on a DAQ and Python
}

\author{
Israel Reyes-Ramírez ${ }^{1}$, Jorge Fonseca-Campos ${ }^{1}$ and Juan Luis Mata-Machuca ${ }^{1}$ \\ ${ }^{1}$ Unidad Profesional Interdisciplinaria en Ingeniería y Tecnologías Avanzadas \\ Instituto Politécnico Nacional \\ Av. IPN 2580, Col. la Laguna Ticomán, GAM, CDMX, C.P. 07340. México. \\ e-mail: ireyesr@ipn.mx, ifonsecac@ipn.mx
}

\begin{abstract}
The renewable energy has grown extensively in the last decades. The photovoltaic panels have been playing a significant role in the technologies used to produce this kind of energy. This tendency is expected to continue in the future, because the cost of the solar panels has been gradually reduced. Systems providing information of the performance of the solar cells can be helpful for technicians and researchers. In this paper a system to measure the I-V curves of photovoltaic cells is presented. This is composed by a data acquisition board (DAQ) and a personal computer (PC). The program to control the DAQ is written in Python. With this system a polycrystalline silicon solar cell was tested under low irradiance conditions (less than $100 \mathrm{~W} / \mathrm{m} 2$ ) with an artificial light source. The repeatability of the $\mathrm{I}-\mathrm{V}$ curves obtained has less than $5 \%$ error.
\end{abstract}

\section{Key words}

Photovoltaic Panels, Python, DAQ, I-V Curves.

\section{Introduction}

The energy is vital for our everyday existence. Recently, the renewable energy coming from sources as the ocean, wind and solar have been promoted to mitigate to some extent the climate change. Among them, the solar energy has attracted much attention, because it is a mature technology, which can be installed almost everywhere. Photovoltaic installations provide the majority of the electricity produced by solar energy. Therefore, to measure their performance is very important. The conversion efficiency of the photovoltaic devices (panels or cells) depends on several factors, such as the irradiance and the temperature. Most commercial solar panels are tested at standard conditions, and the electric characteristics found in their datasheets refer to this condition. However, the photovoltaic devices are installed in places where this condition does not hold. Thus, the measurement of the I-V curve gives us a better knowledge of the electrical characteristics of the solar photovoltaic devices under different scenarios.

There are several methods reported to measure the I-V curves of solar cells, such as variable resistor, capacitive load, electronic load, bipolar power amplifier, fourquadrant power supply and the DC-DC converter [1]. In general, the measurement of the I-V curve is realized by controlling the current supplied by the photovoltaic device in the region where the current reaches zero, and the voltage reaches zero. The variable resistance circuit is the simplest. Although, it only can be applied to low power modules, because it requires resistors of very high power. The method of bipolar power amplifier allows to measure the dark I-V curve, because the current and the voltage in the photovoltaic device can be reversed. Solar photovoltaic modules of medium power can be measured with this method. Meekhun et al. [2] reported an alternative circuit, which can be considered as a hybrid of the above methods, but it employs operational amplifiers instead of transistors.

The minimum experimental set up to measure the I$\mathrm{V}$ curves of solar cells requires at least of a voltage source, a voltmeter and an ammeter. Some low cost systems have been proposed to solve this problem [2-7]. These are based on microcontrollers and DAQ's. Devices such as the microcontrollers have analog inputs, but do not have analog outputs. Therefore, strategies to supply an external voltage to the solar cell must be applied. Some researches solve this problem with an external AC power supply with DC offset adjustment [3]. Others employ a pulse width modulated signal and one external circuit acting as a digital to analog converter [4]. Unfortunately, these strategies make the system less portable and more complex.

Most DAQ's have several analog inputs and a few analog outputs. Thus, these are adequate to measure the electrical characteristics of the photovoltaic cells [2,5-7]. However, the voltage levels supplied by these devices goes from 0 to $5 \mathrm{~V}$, which are insufficient to measure the $\mathrm{I}-\mathrm{V}$ curves in all the quadrants. External circuits must be employed to solve this problem. The systems reported in [2-7] are controlled with commercial software as LabVIEW or Matlab. Due to the software license cost, these devices become expensive. An alternative is to use an open source programming language as Python.

In this work, a system to measure the electric characteristics of a solar cell is presented. It is based on a low cost DAQ of National Instruments (NI-6009), one external circuit (voltage level adapter) and one program written in Python. With this system one polycrystalline silicon solar cell was tested under low irradiance conditions (less than $100 \mathrm{~W} / \mathrm{m} 2$ ) with an artificial light source (tungsten light source). 


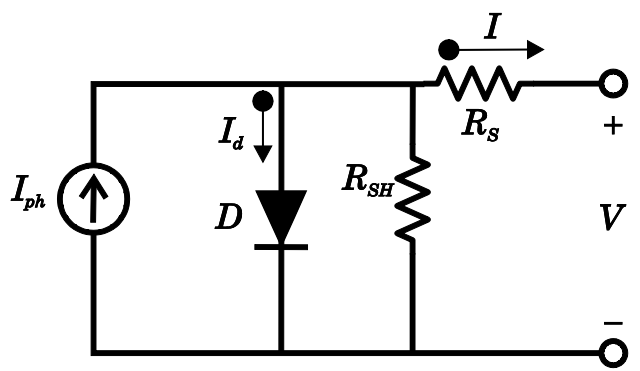

Fig. 1. Single-diode solar cell electric model.

\section{Solar Cell Model}

Equivalent electric circuits are usually used to describe the behavior of the solar panels. The most common models explained in the literature are the singlediode and the two-diode model [8]. The equivalent circuit of the simplified single-diode model is shown in the Fig. 1. The behavior of the current is nonlinear and is given by,

$$
I=I_{p h}-I_{0}\left(e^{\frac{V+I R_{S}}{n_{S} V_{T}}}-1\right)-\frac{V+I R_{S}}{R_{S H}},
$$

where $I$ is the photovoltaic cell current, $I_{p h}$ stands for the photocurrent, $I_{0}$ is the diode saturation current, $V$ stands for the photovoltaic cell voltage, $R_{S}$ is the series resistance of the cell, $n_{S}$ is the number of cells in series, $v_{T}$ is the thermal voltage equivalent and $R_{S H}$ stands for the internal shunt resistance.

The illuminated I-V curve extends to three quadrants (I, II and IV). In the first quadrant $V>0$ and $I>0$. In the fourth quadrant $V>0$ and $I<0$. The inverse of the slope of the straight line section of the curve, which goes from the fourth quadrant to the first quadrant is associated to $R_{S}$ by the eq. 2 [2]

$$
R_{S}=\frac{\Delta V_{1}}{\Delta I_{1}}
$$

where $\Delta V_{1}$ and $\Delta I_{1}$ are measured in the region near to the point at which the current of the I-V curve crosses the voltage axis $\left(V_{O C}\right)$. In the second quadrant $V<0$ and $I>0$. The inverse of the slope of the straight line section of the curve, which goes from the second quadrant to the first quadrant is associated to $R_{S H}$ by the eq. 3 .

$$
R_{S H}=\frac{\Delta V_{2}}{\Delta I_{2}},
$$

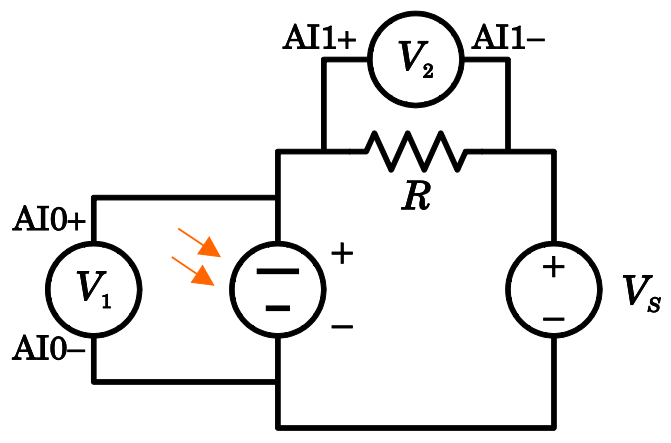

Fig. 2. Circuit used to measure the I-V curve where $\Delta V_{2}$ and $\Delta I_{2}$ are measured in the region near to the short circuit current $I_{S C}$.

\section{System Description}

\section{A.- Electric Circuit}

The diagram of the electric circuit used to measure the I-V curve is shown in the Fig 2. During the preliminary experiments the analog output of the DAQ was connected as the voltage source $V_{S}$. However, the $I_{S C}$ could not be reached, because the output voltage provided by the DAQ was always positive in the range of 0 to $5 \mathrm{~V}$. This is the same inconvenient reported in [5]. By this reason, the voltage level adapter circuit proposed in [2] was implemented. This circuit with the final values of the components used during the experiments is shown in the fig. 3. Once a negative offset was introduced in the analog output with the above circuit, the voltage and the current across the solar cell were measured. The configuration of the analog input channels of the DAQ were chosen as differentials. In the Fig. $2 \mathrm{AIO}+$ refers to

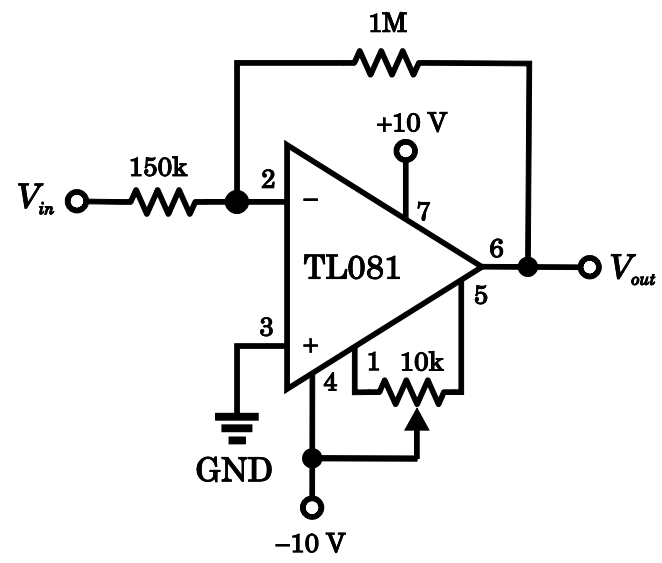

Fig. 3. Voltage adapter circuit.

the positive terminal of the first analog input channel and AIO - is the negative pin of this channel. An external resistor $R$ was used to monitor the current as it is shown in the Fig. 2. The polarity of the second analog input channel is shown in the same figure.

\section{B.- Pyhton Program}

The program was written in Python 3.5 and it has approximately 200 lines of code. It can be requested by email to the authors. PyDAQmx's module must be installed in order to control the NI-DAQ with Python [8]. A flowchart of program is shown in the Fig. 4. When National Instruments DAQ's are controlled by text based programming environments, it is required to configure the DAQ following the next steps: create a task and virtual channels, configure the timing parameters, start the task, perform a read operation from the DAQ, perform a write operation to the DAQ and stop and clear the task. These steps correspond to the first three blocks of Fig. 4. The last two blocks correspond to data analysis and data visualization. 


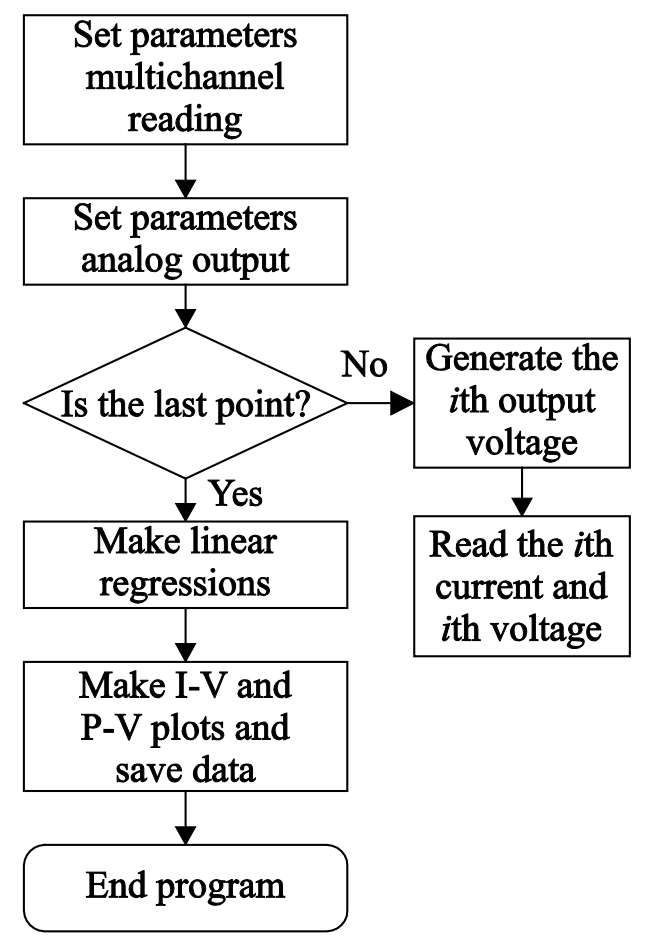

Fig. 4. Flowchart Python program.

\section{C.-Experimental Setup}

Only one commercial polycrystalline silicon solar cell was tested. The nominal voltage of operation of the cell is $2 \mathrm{~V}$ and the maximum current provided is $75 \mathrm{~mA}$. It has an area of $25 \mathrm{~cm}^{2}$. As it was mentioned above, the DAQ and the external circuit were used to generate the voltage in the cell test circuit, as well as the monitoring of the voltage and the current across the solar cell. In all experiments one tungsten light source with a nominal electric power of $150 \mathrm{~W}$ was employed. The normalized spectrum of this optical source is shown in the Fig. 5. As it can be seen from Fig. 5, the light source ranges from the visible to the near infrared and it has a small dip around 850nm. The optical power was measured with the commercial optical multimeter from Newport, model 1830 C, along with the photodetector model 918D-IS-1 with a range from 400 a $1650 \mathrm{~nm}$.

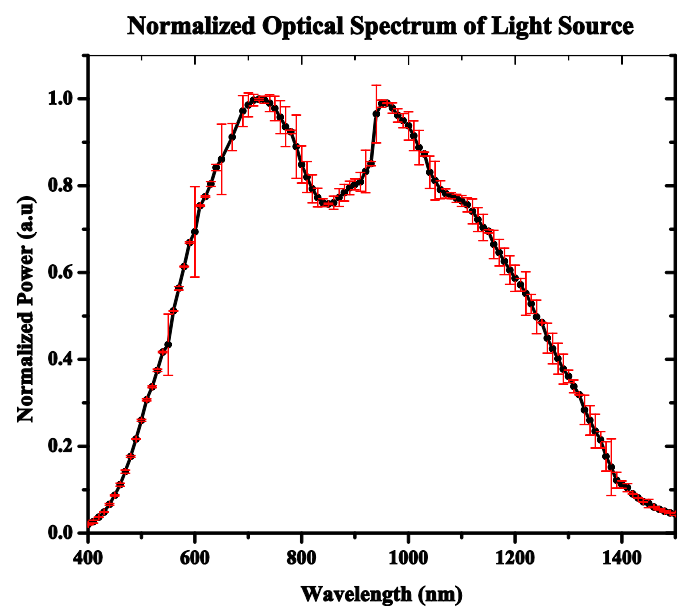

Fig. 5. Spectrum of light source.

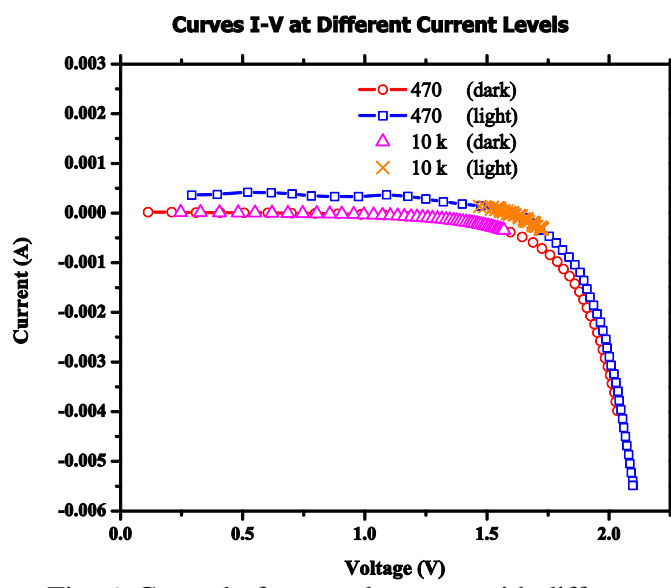

Fig. 6. Control of external current with different resistors.

\section{Results and Discussion}

\section{A.- Control of the current of the test circuit}

Several external resistors were tested to control the external current provided with the test circuit of Fig. 2. In the Fig. 6 four I-V curves in the dark and under illumination are shown. Two were measured with a resistor of $10 \mathrm{k} \Omega$, the rest were taken with a resistor of $470 \Omega$. It can be seen from this figure that an increase of the external resistor reduces the range of voltages measured in the I-V curve (see triangles and cross symbols). A decrease in the external resistor increases the range of the voltages measured in the I-V curve. These measurements were realized without the voltage adapter circuit. Thus, the short circuit current was not reached. When the external circuit was used in the experiments, it was necessary to reduce the value of the external resistor. A $2 \mathrm{~W}$ power resistor of $47 \Omega$ was used in the rest of the experiments. Under these conditions the experimental points of the I-V curves crossed the first, the second and the fourth quadrant at irradiances up to $85 \mathrm{~W} / \mathrm{m}^{2}$. A shift to right of the I-V curve can be seen in the Fig. 6, when the cell is illuminated.

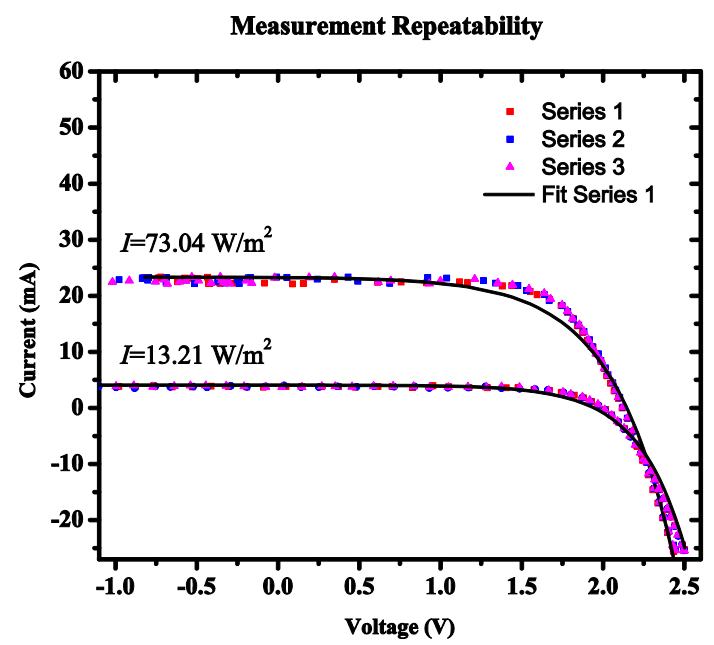

Fig. 7. I-V Curves at different irradiances. 


\section{A. Curves repeatability}

In any system is very important to have the possibility to repeat the measurements with a small error. In the Fig. 7 are shown six measurements of the I-V curve at two different irradiances. As expected, when the irradiance is increased the available power is increased. On the other hand, the comparison of the experimental points of different curves is difficult, but comparing fitting parameters becomes easier. By this reason the exponential fitting of the eq. 4 was realized.

$$
I=A_{1} \exp \left(-V / t_{1}\right)+y_{0}
$$

The fitted equation of the experimental points is shown with the solid line in Fig. 7. The results of fitting are better for small irradiances. The fitting coefficients $A_{1}$, $t_{1}, y_{0}$ and the correlation coefficient $r$ with their error are shown in the Table I. For the great majority of the coefficients its error is less than $1.5 \%$. However, for the coefficient $A_{1}$, when $I=73.04 \mathrm{~W} / \mathrm{m}^{2}$, its error is $\sim 4.6 \%$.

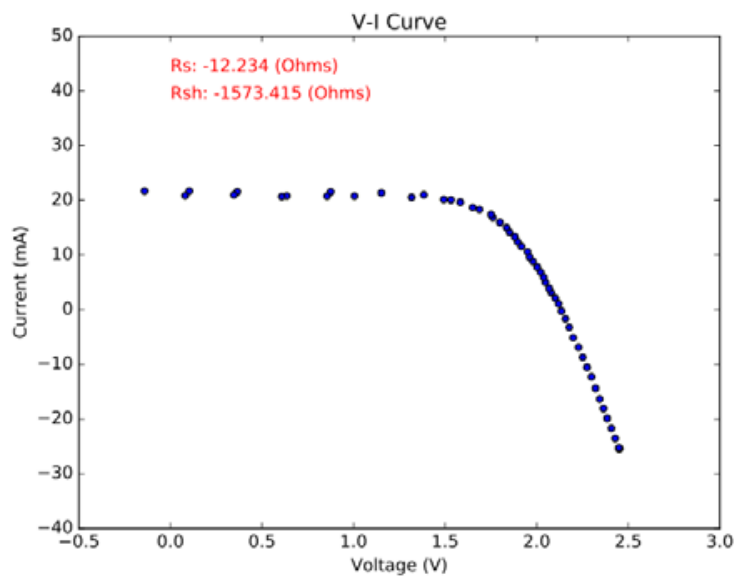

Fig. 8. Curve generated by the Python program

The coefficient $y_{0}$ of the fitted curve can be used as an estimation of the short circuit current. A value of $\overline{y_{0}}=4.038 \mathrm{~A}$ was obtained when the cell was irradiated with $13.21 \mathrm{~W} / \mathrm{m}^{2}$. At $I=73.04 \mathrm{~W} / \mathrm{m}^{2}$ this coefficient was $\overline{y_{0}}=23.378 \mathrm{~A}$. Both values are approximate to the value of current when $V \approx 0$, as it can be seen in the Fig. 7.

\section{C.- Program Output}

In the Fig. 8 is shown the plot of the I-V curve generated with the program. As it can be seen from this figure, this time the curve crosses the three quadrants (I, II and IV). Because, the external circuit was employed. Due to this situation the short circuit current and the open circuit voltage can be determined.

An estimation of $R_{S}$ and $R_{S H}$ was obtained with a linear regression. See eqs. 2 and 3 . These values are displayed in the fig. 8. The value of $R_{S}$ is small and the value of $R_{S H}$ is big. Unfortunately, in different tests these values were changing. Therefore, it is necessary to improve their calculation.

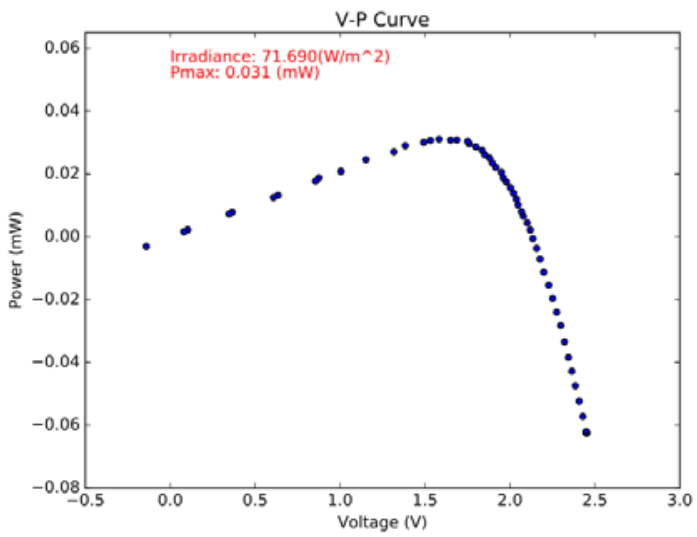

Fig. 9. P-V Curve.

Once that the I-V curve has been measured it is natural to get the power-voltage curve $(\mathrm{P}-\mathrm{V})$. In the Fig. 9 is shown this curve. In this plot the maximum power and the irradiance are displayed.

The program also creates two files having the values of the current, the voltages, the irradiance, the shunt resistor and the series resistor.

\section{Conclusions}

6.

The electrical characteristics of a polycrystalline silicon solar cell was tested under low irradiance conditions with a low cost system.

The system is controlled with Python, which is an open source software.

The measurements can be repeated with a small error.

\section{Acknowledgments}

The authors want to thank to the Instituto Politécnico Nacional (IPN), COFAA-IPN and EDI-IPN for the financial support provided to do this work. Also, the first author wants to thank to CONACyT-México.

\section{References}

[1] E. Duran, M. Piliougine, M. Sidrach-de-Cardona, J. Galan \& J.M. Andujar, "Different methods to obtain The I-V curve of PV modules: A review," in Conference Record of the IEEE Photovoltaic Specialists Conference, pp. 1-6, 2008.

[2] D. Meekhun, Vincent B., J. M. Dilhac \& G. Blin, "An automated and economic system for measuring of the current-voltage characteristics of photovoltaic cells and modules," in International Conference on Sustainable Energy Technologies IEEE, pp. 144 148, 2008

[3] Y. Atia, M. Zahran, A. Al-Hossain, "Solar cell curves measurement based on LabVIEW microcontroller interfacing," in Proceedings of the 12th WSEAS International'Conference on Automatic Control, Modelling \& Simulation, pp. 59-64, 2010.

[4] S. Sousa, M. Onofre, Tiago Antunes, Cláudio Branco, José Maia ,J. I. Rocha \& V. F. Pires, "Implementation of a low cost data acquisition board for photovoltaic arrays analysis and diagnostic,” in 
International Conference on Renewable Energy Research and Applications ICRERA, pp. 1084 - 1088, 2013.

[5] E. A. Mayer \& A. L. Powell, “A Low-cost laboratory experiment to generate the I-V characteristic curves of a solar cell," Annual Conference \& Exposition of the American Society for Engineering Education (ASEE), pp. 1-13, 2011.

[6] Ch. B. Ahmed, M. Kassas \& S. E. Ahmeda, "PVstandalone monitoring system performance using LabVIEW," International Journal of Smart Grid and Clean Energy, pp. 44-50, 2014.

[7] Y. Yadav, R. Roshan, S. Umashankar, D. Vijayakumar \& D. P. Kothari, "Real time simulation of solar photovoltaic module using LabVIEW data acquisition card," in International Conference on Energy Efficient Technologies for Sustainability (ICEETS), pp. 512 - 523, 2013.

[8] V. Tamrakar, S.C. Gupta \& Y. Sawle, "Single-diode and two-diode PV cell modeling using Matlab for studying characteristics of solar cell under varying conditions," Electrical \& Computer Engineering: An International Journal (ECIJ), Vol. 4, pp. 67-77, 2015.

[9] PyDAQmx : a Python interface to the National Instruments DAQmx driver, Pierre CLADÉ. Available at http://pythonhosted.org/PyDAQmx/ 